\title{
HEPARIN AND OTHER SULFATED POLYANIONS: THEIR INTERACTION WITH THE BLOOD PLATELET *
}

\author{
M. Lois Tiffany and John A. Penner \\ Simpson Memorial Research Institute \\ University of Michigan \\ Ann Arbor, Michigan 48109
}

\section{INTRODUCTION}

Heparin is a heterogeneous mixture of partially sulfated polysaccharide chains. Different subfractions of heparin have been found to interact with platelets to different degrees, which do not parallel the anticoagulant activity of the preparation.' There is some suggestion that the low antithrombin III affinity of a subfraction results in more potent platelet interaction. It is, however, extremely difficult to relate function to molecular structure when heterogeneity of molecular species exists. A logical approach is to work with more homogeneous model compounds (sulfated polyanions) in order to understand the molecular features involved in the functions observed and is the basis for the following report.

\section{Materials AND METhODS}

The following materials were used: heparin beef lung and intestinal mucosa, Upjohn Company, Kalamazoo, MI and Armour Pharmaceutical Company, Phoenix, AZ; polyvinyl sulfate (molecular weight 100,000), dextran sulfate (molecular weight 500,000 and 40,000 ), dextran (molecular weight 500,000 and 10,000) and carrageenan (lamda and iota type), Sigma Chemical Company, St. Louis, MO; polyanetholesulfonate, Eastman Kodak Company, Rochester, NY and Roche Diagnostics, Nutley, NJ; cellulose sulfate, Aldrich Chemical Company, Milwaukee, WI; poly methacrylic acid, Polysciences, Warrington, PA; poly-L-glutamic acid, Pilot Chemicals, Inc., Watertown, MA.

Blood was collected in buffered citrate, monojet tubes, final concentrated citrate $0.38 \%$ and centrifuged $(160 \times g, 5 \mathrm{~min})$ to prepare platelet rich plasma. Platelet poor plasma was made by spinning at $1000 \times g, 10 \mathrm{~min}$. The platelet rich plasma count was adjusted to be 200,000 with plasma or phosphate buffered saline, as desired. To remove plasma as completely as possible, the procedure of albumin density centrifugation followed by Sepharose 2B was followed. ${ }^{2}$

Comparison aggregation studies were using a dual sample aggregometer, Scienco Inc., Morrison, $\mathrm{CO}$. Aggregation with release measured simultaneously was recorded with a lumiaggregometer, Chrono-log Company, Havertown, PA. All polyanions were diluted in phosphate-buffered saline, pH 7.8 and $0.01 \mathrm{ml}$ was added to plasma for pre-incubation studies. This was then added to $0.2 \mathrm{ml}$ of platelet-rich plasma $\left(4 \times 10^{5}\right.$ platelets per $\mu$ l) and aggregation was recorded at $37^{\circ} \mathrm{C}$.

* Supported by The Skillman Foundation, Detroit, Michigan. 


\section{RESUlts}

Studies discussed in the following portion of the paper are illustrated in FIGUREs 1 through 6 which demonstrate the effects of sulfated and nonsulfated polyanions on platelet aggregation under conditions specified.

\section{Discussion}

Some insight into the molecular properties required for activation of platelets is derived by our comparative studies of the various polyanions. Dextran sulfate of molecular weight 500,000 was more effective than dextran sulfate of molecular weight 40,000 (FIG. 5C). The degree of sulfation is stated by the manufacturer to be the same. Therefore, chain length is an important factor.

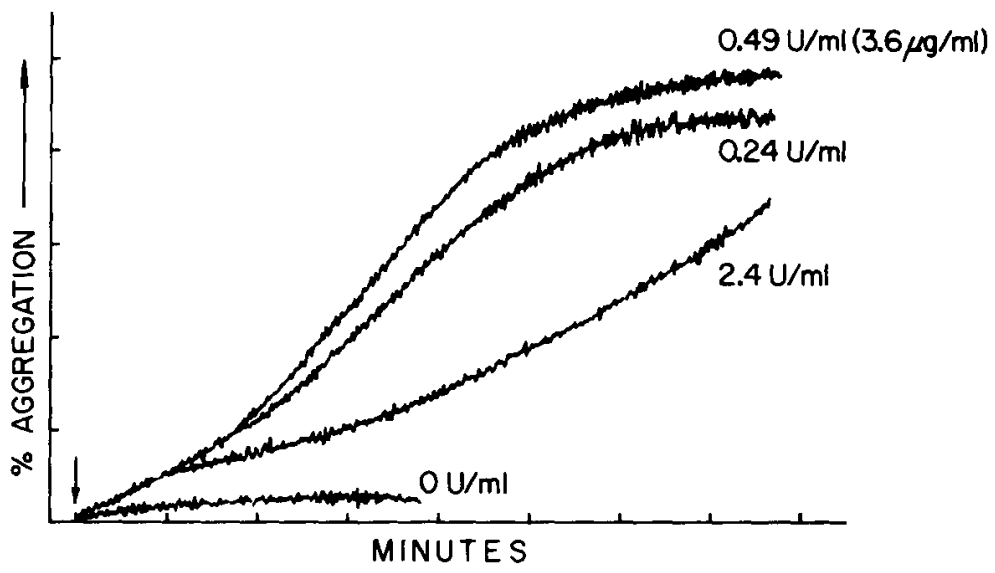

Figure 1. Aggregation induced by heparin.

Dextran, sulfated and nonsulfated, of the same molecular weight, 500,000, also were compared. No platelet aggregation was possible with any concentration of dextran when the conditions of observation were $37^{\circ} \mathrm{C}$ with stirring. If, however, the conditions were room temperature and no stirring, platelets clumped in a high concentration of both forms of dextran $(5 \%)$. The clumps were easily dispersed by shaking. This is similar to the results reported by Taketomi and Kuramoto. ${ }^{3}$ The same percent dextran $(5 \%)$ completely inhibited platelet aggregation by ADP, epinephrine and collagen, as did similar concentrations of the sulfated polyanions. This inhibition may be a result of coating of the platelet with polymers leading to nonspecific interference with activation of the platelet.

High concentration of polymethacrylic acid $(25 \mu \mathrm{g} / \mathrm{ml})$ was found to induce a slight platelet aggregation response, but another polyanion, poly-Lglutamic acid was without any activity. Thus, a negative charge on the polymer is a necessary, but not a sufficient condition, for activation.

All of the sulfated polyanions studied could induce some platelet aggrega- 


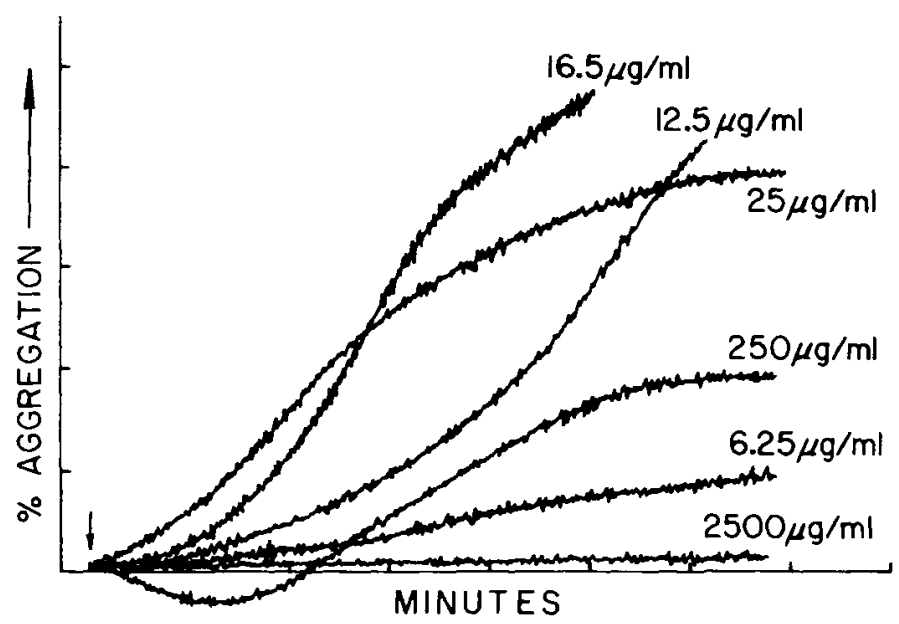

FIGURE 2. Aggregation induced by polyvinyl sulfate.

tion (examples of aggregation studies are given in FIGs. 1, 2, $3 \& 4$ ), although only over a limited range of concentrations and in the presence of plasma or serum (example, Fig. 6A). Preincubation of the polyanion either in plasma or in heat-denatured serum enhanced the platelet response (FIG. 6B) suggesting an indirect mechanism of platelet aggregation mediated by a plasma factor. There are a number of reports in the literature which would support a concept that the protease precursor, plasminogen, is the plasma factor responsible. The possible interaction of the sulfated polyanion heparin with an inhibitor to plasminogen activator (thus accelerating plasminogen activation) was postulated by Vairel." Similarly, a "release" of plasminogen activator by sulfated polyanions was suggested by Olesen ${ }^{5}$ and Ugar and Mist. ${ }^{6}$ On the other hand,

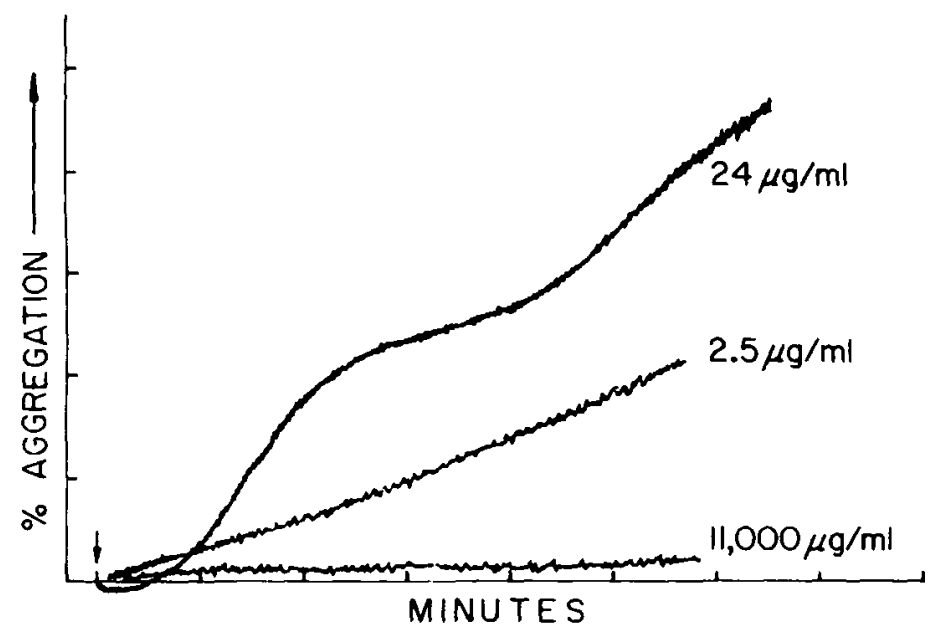

FIoure 3. Aggregation induced by dextran sulfate. 
Figure 4. Aggregation with concomitant ATP release by relatively high concentration of polyanethol sulfonate $(25 \mu \mathrm{g} / \mathrm{ml})$. Aspirin is not very effective in inhibiting aggregation or release.
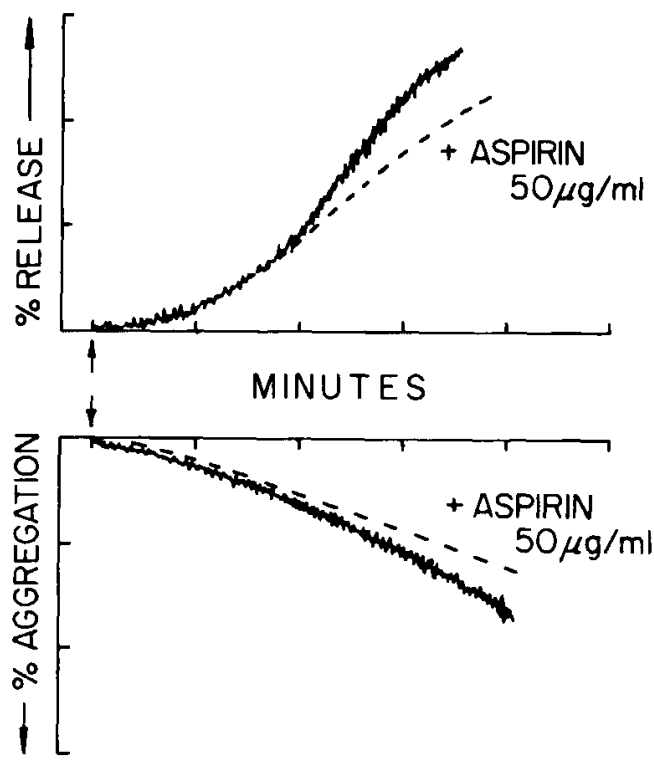

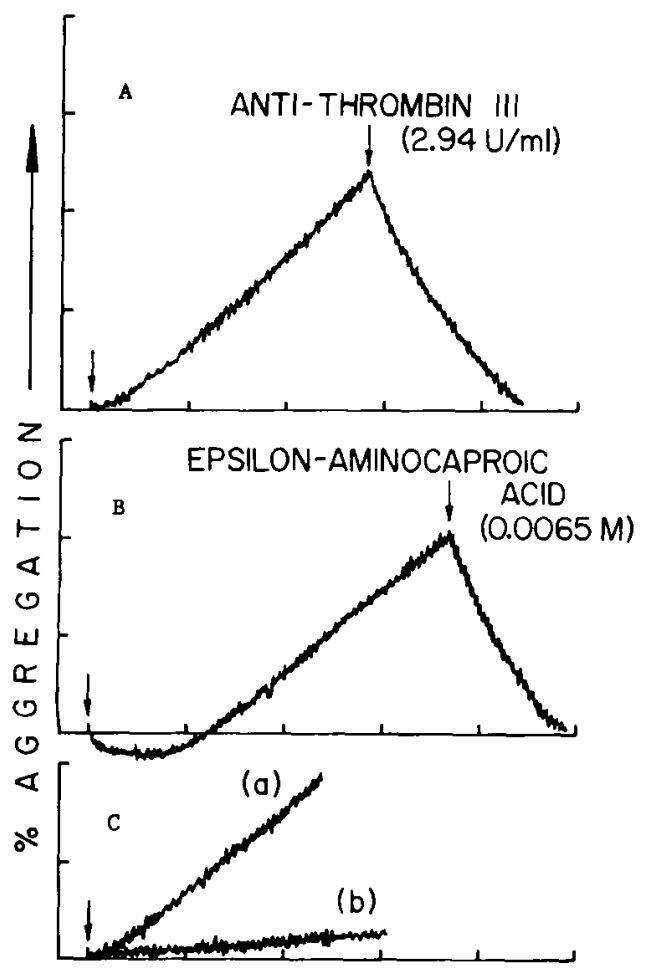

Figure 5. (A) Aggregation by $25 \mu \mathrm{g} / \mathrm{ml}$ polyvinyl sulfate is rapidly inhibited and completely reversed by $2.94 \mathrm{U} / \mathrm{ml}$ of antithrombin III. (B) Similar inhibition and reversal of aggregation produced by 25 $\mu \mathrm{g} / \mathrm{ml}$ of cellulose sulfate is seen with epsilon aminocaproic acid at 0.0065 M. (C) Aggregation is produced at low concentrations with (a) $25 \mu \mathrm{g} / \mathrm{ml}$ of dextran sulfate 500,000 compared to (b) the minimal response by dextran 40,000 under the same conditions of testing.

MINUTES 
heparin also was found to accelerate the rate of formation of a complex of antithrombin III with plasmin. ${ }^{7}$ This would increase the rate of inhibition of proteolytic and esterolytic activity of this protease. It is possible that low concentrations of heparin increase the formation of plasmin, while excess heparin would interfere with the activity of this enzyme once it is formed. If plasmin is responsible for platelet aggregation produced when polyanions are present, an optimal concentration of polyanion would, therefore, be expected to be most effective in inducing aggregation.

Inhibition of aggregation by the sulfated polyanions occurred at low concentrations of the plasmin inhibitor antithrombin III $(2.94 \mathrm{u} / \mathrm{ml}$ ) (FIG. 5A), as
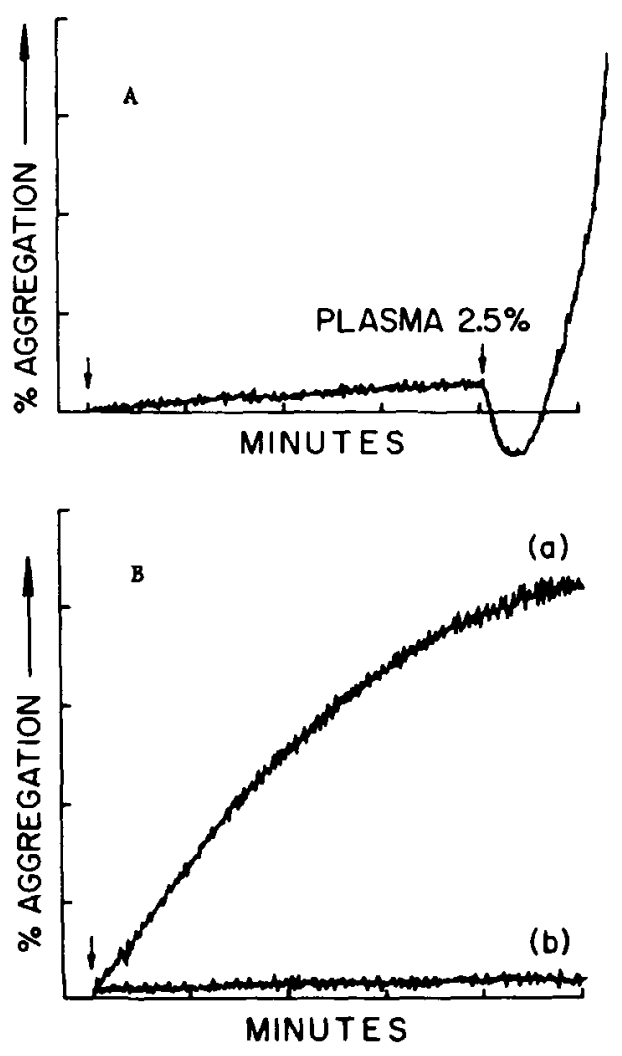

Figure 6. (A) Platelets, well washed by albumin density centrifugation followed by gel filtration are not aggregated by $\lambda$ carrageen ( $50 \mu \mathrm{g} / \mathrm{ml}$ ) until plasma is added, to be $2.5 \%$ of final volume. (B) In (a) threshold concentration for aggregation by polyvinyl sulfate exceeds $2.5 \mu \mathrm{g} / \mathrm{ml}$ when the polyanion is first preincubated in plasma or serum for $25 \mathrm{~min}$ at room temperature. In control (b) with no preincubation, there is no aggregation response in the first 5 min in contact with the platelets at $37^{\circ} \mathrm{C}$.

well as with $\mathrm{Cl}$ esterase inhibitor (not shown) and also occurred with low concentration of epsilon aminocaproic acid $(0.0065 \mathrm{M}$ ) (FIG. 5B), an inhibitor of plasminogen activation. ${ }^{8, \theta}$ These findings also may be taken as evidence to support the concept that activated plasminogen is capable of inducing platelet aggregation and that the sulfated polyanions exert their action by means of this protease.

In summary, we find sulfated polyanions induce platelet aggregation indirectly by activation of a plasma factor. On a weight basis, the amount of polyanion needed to induce threshold aggregation, is a function of the degree 
of polymerization and the degree of sulfation of the polymer. Negative charge on the polymer is a necessary but not sufficient condition for activation. Platelet aggregation is inhibited by high concentration (5\%) of all poly-saccharides whether they are sulfated or not.

\section{REFERENCES}

1. Salzman, E. W., R. D. Rosenberg, M. H. Smith, J. N. Lindon \& L. Favreau. 1980. Effect of heparin and heparin fractions on platelet aggregation. J. Clin. Invest. 65: 64-73.

2. Timmons, S. \& J. HAwiger. 1978. Separation of human platelets from plasma proteins including Factor VIII:VWF. Thromb. Res. 12: 297-306.

3. TAKETOM, Y. \& A. KURAMoto. 1978. Ultrastructural studies on the surface coat of human platelet aggregated by poly lysine and dextran. Thrombos. Haemostas. (Stuttg.) 40: 11-23.

4. VAIREL, E. G. 1975. Simple in vitro method for screening fibrinolytic substances. In Progress Chemical Fibrinolysis and Thrombolysis, Vol. 2, J. F. Davidson, M. M. Samama \& P. C. Desnyers, Eds.: 271-277. Raven Press, New York.

5. OzESEN, E. S. 1961. A fibrinolytic system in human plasma activated by peptone or acid polysaccharides. Scand. J. Clin. Lab. Invest. 13: 410-415.

6. UNGAR, G. \& S. H. Mist. 1949. Observations on the release of serum fibrinolysin by specific antigen, peptone, and certain polysaccharides. J. Exp. Med. 90: 39-51.

7. Highsmith, R. F. \& D. Rosenberg. 1974. The inhibition of human plasmin by human antithrombin-heparin cofactor. J. Biol. Chem. 249: $4335-4338$.

8. MARTWARDT, F. 1978. Naturally occurring inhibitors of fibrinolysis. In Fibrinolytics and Antifibrinolytics. Editor F. Markwardt, Springer-Verlag. Berlin, Heidelberg, New York.

9. Alraersio, N., A. P. Fletcher \& S. Sherry. 1959. Aminocaproic acid: An inhibitor of human plasminogen. J. Biol. Chem. 234: 832-837. 